\title{
A Conversation Between Michael Phelps and Jason Lewis
}

\author{
Michael E. Phelps ${ }^{1}$ and Jason S. Lewis ${ }^{2}$ \\ ${ }^{I}$ Department of Molecular and Medical Pharmacology, David Geffen School of Medicine at UCLA, Los Angeles, California; and \\ ${ }^{2}$ Memorial Sloan Kettering Cancer Center, New York, New York
}

$\mathbf{J}$ ason S. Lewis, PhD, Emily Tow Jackson Chair in Oncology at the Memorial Sloan Kettering Cancer Center (New York, NY), spoke with Norton Simon Professor and Chair of the University of California at Los Angeles Department of Molecular and Medical Pharmacology and Director of the Crump Institute for Molecular Imaging Michael Phelps. Phelps is known for his invention of the PET scanner with his postdoc at the time, Edward Hoffman; graduate student, Henry Huang; and electrical engineer, Nizar Mullani, at the Mallinckrodt Institute of Radiology at Washington University in the early 1970s. Phelps was born in Cleveland, OH, but grew up on the Olympic Peninsula in Washington state. He earned his BS in chemistry and mathematics from Western Washington State University in 1965 and his $\mathrm{PhD}$ in chemistry from Washington University in St. Louis in 1970 . He joined the faculty of the Washington University School of Medicine in 1970. In 1975 and 1976, Phelps was a member of the faculty at the University of Pennsylvania in Philadelphia. In 1976, he was recruited to the David Geffen School of Medicine at UCLA. He has published 720 peer-reviewed articles and 4 textbooks and has been the PI of $\$ 245$ million in grants and funding. He has received awards such as the Georg Charles de Hevesy Nuclear Pioneer award (from SNMMI) and the Georg von Hevesy award (from the von Hevesy Foundation in Zürich, Switzerland); chaired the 1983 Nobel Symposium in Stockholm, Sweden; received the S. Weir Mitchell Award, Academy of Neurology; gave the keynote address at the 2007 Nobel Symposium in Stockholm; and received the Richard and Hinda Rosenthal Award from the American College of Physicians, the Enrico Fermi Presidential Award from President Bill Clinton, the Charles F. Kettering Prize from the General Motors Cancer Research Foundation, and others. He is a member of the National Academy of Sciences and the National Academy of Medicine and was 1 of the 3 founders of CTI Molecular Imaging, later acquired by Siemens. His wife, Patricia Phelps, is a professor of Integrative Biology and Physiology at UCLA. They have 2 children: Patrick Phelps and Katy Phelps.

Dr. Lewis: Thank you for taking the time to talk today. I want to start out with a little on your background information and personal history. How did you get into science and how did you become one of the "fathers of PET"?

Dr. Phelps: I was raised in a poor Irish community in the northwest part of Washington state. Boys were encouraged to become boxers or priests. I spent time as a boxer and went to seminary for $6 \mathrm{mo}$. A severe head injury from a car accident left me in a coma, so I had to take a different direction. I went to college and decided to go into math and chemistry, although I

COPYRIGHT @ 2019 by the Society of Nuclear Medicine and Molecular Imaging. really didn't know much about them. But the Irish have a saying: "fake it until you can make it." In the beginning, I pretended that I liked math and chemistry-until the time came when I did. I went to graduate school in chemistry at Washington University and, when I got my PhD, had offers to join the faculty at the Massachusetts Institute of Technology and the Max Planck Institute in Germany. I decided to accept a faculty position at the Washington University School of Medicine-not

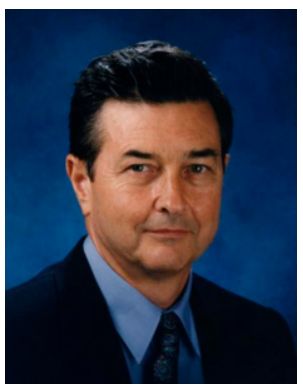

Michael E. Phelps, PhD

At Washington University I had to teach medical students about the biochemistry of disease. At that time, the study of biochemistry in patients was limited to samples of blood, urine, and biopsy/surgical samples. So, I decided to explore ways to examine the biochemistry of disease in patients through imaging.

Dr. Lewis: It is well known that you have a background in boxing. Did that help shape who you are professionally now?

Dr. Phelps: Absolutely! Boxers are trained to not focus on winning fights but to focus on winning rounds to win fights round by round. You must accept that you win some rounds and you lose some. When you lose rounds you are trained to have the will and courage to not lose the next. Boxers are trained that they must be willing to get hurt to have the opportunity to win and that training is a way of becoming, whereas fighting is about standing and delivering when the time comes. This is as much about life as it is about boxing.

Dr. Lewis: Yes, that's life.

Dr. Phelps: I teach these principles to students as they go through their developmental years of becoming basic or clinical scientists.

Dr. Lewis: You mentioned students. You are a mentor to many of us in the field, me included, but who do you consider the best mentor you've ever had?

Dr. Phelps: Well, ironically, there are 2 and neither is from academia. One was my boxing coach, Ira Goldberg. When our house burned down, killing my 2 younger brothers and severely burning my mother, Ira became my boxing coach, my teacher, and my friend.

The other was Norton Simon. He created the greatest personal, corporate, and foundation wealth in our country after the Great Depression and, by himself, collected $\sim \$ 3$ billion in art from the Old Masters, when even the Getty Museum with its enormous wealth couldn't achieve this. I was with him almost every day from 1980 until his death in 1993, either on the phone or in person. Norton had a wonderful approach to life. He said, "There's a natural curve in life. You go up, plateau, and go down, and the 
only way to deal with this is by starting new curves and, by this, to remain in a state of becoming."

Dr. Lewis: Do you think these 2 people have become central to your own philosophy of mentoring?

Dr. Phelps: Yes. They became part of me, and their teachings were put into perpetuity by my teaching to others, who pass these teachings on through the generations that follow. This evolved in many different ways. Sometimes I tell others quotes from Ira or Norton that they didn't actually say, but it's what they taught me, now translated into my teachings so that it has become a mix of them and me-but mostly them.

Dr. Lewis: So, getting back to science. You said you went into imaging because you wanted to provide access to biochemistry of disease in patients. You've been the chair of a pharmacology department for many years. What is nuclear medicine's most important application? Is it in drug design? Is it in diagnosis and treatment of disease or all of the above? Where do you see the most important link between nuclear medicine and pharmacology?

Dr. Phelps: Nuclear medicine and molecular imaging assays are based on the biochemistry and biology of disease and should be integrated and paired with molecular- and cell-based treatments of disease. I implemented this integration by moving nuclear medicine and PET/CT from radiology into pharmacology. There are always stories behind the things that happen in life. The dean of the UCLA medical school at the time, Sherman Mellinkoff, did something remarkable. He recruited me from Penn and, in 1984, appointed me as chief of the Division of Nuclear Medicine in the Department of Radiology, which was coupled to a large PET
Dr. Lewis: What do you think is professionally your biggest disappointment? Where did you not succeed?

Dr. Phelps: Oh my god! I'm the master of mistakes. But that's where the rules of boxing help in winning and losing rounds and sustaining the will to keep doing your rounds. Through all these rounds, learning must remain as a way of living. When Norton Simon was dying, I sat on his bed and asked "What do you want to be written about you after you die?" He said "What do you think?" Ill prepared for such a profound question, I said "How much you accomplished so quickly?" He looked at me and said, "But that's not true! You are not taking into account all the years of struggle with failures, successes, and mistakes before I moved quickly to an outcome. One must go through the struggles of these earlier times and avoid temptations to do lesser things or things for the wrong reasons. You need to teach your students about these early times and the reality of what is required to be successful, not just about the times of success."

Dr. Lewis: I was looking at one of your earliest papers in 1973-so cast your mind back. It's on in vivo measurement of cerebral glucose metabolism using ${ }^{11}$ C-labeled glucose with Raichle, Grubb, Welch, Ter-Pogossian, etc. Does it surprise you that metabolic imaging is making such a comeback for so many indications almost 50 y later?

Dr. Phelps: No, not at all. Metabolism is fundamental to how the cells of our bodies function in health and disease, and there is no metabolism more fundamental in the body than glucose metabolism. From glucose and 9 amino acids from our diet, every

"Norton had a wonderful approach to life. He said, 'There's a natural curve in life. You go up, plateau, and go down, and the only way to deal with this is by starting new curves and, by this, to remain in a state of becoming."'

molecular imaging research program I had established with my colleagues and students. Sherm did this because I convinced him that I needed a clinical practice and a board-certified training program to make PET a clinical reality.

Dr. Lewis: What do you consider your greatest achievement professionally, post the invention of PET? Is it, for example, your pivotal role in getting U.S. Food and Drug Administration (FDA) approval and reimbursement for ${ }^{18} F-F D G$ ? Or is it something else?

Dr. Phelps: Well, I think there were many different things. Ed, Henry, Nizar, and I could never have imagined when we invented the PET scanner what people throughout the world would come to accomplish in discoveries with PET and the influence that it would have on medicine. I am sure the same is true when Lou Sokoloff developed the ${ }^{14} \mathrm{C}$-deoxyglucose tracer kinetic model for autoradiography and when Joanna Fowler and Al Wolf synthesized ${ }^{18} \mathrm{~F}$-2-fluoro-2-deoxy-D-glucose (FDG). Regarding FDA approval, reimbursement, and medical adoption, great faculty in medical schools across America came together to get this done, not industry, and, of course, we were helped and supported by U.S. Senators Ted Stevens and Ted Kennedy, who were loyal and trusting friends who believed in us and in the value PET provides to science and medicine. compound in the body can be synthesized. Overall, in separating health and disease, nothing is more important than glucose metabolism. Of course, there are many specific questions in health and disease that require specific imaging probes and the assays they provide.

There's a very important lesson to be learned from ${ }^{11} \mathrm{C}$-labeled glucose vs. FDG. In the early times of biochemistry, substrate analogs were developed to isolate steps in complex biochemical reaction sequences, so they could measure those steps accurately. This is where DG and FDG originated, as biochemists developed glucose analogs to isolate facilitated transport and the subsequent enzyme-mediated metabolism of glucose. Their rigorous kinetic measurements demonstrated that only the substitution of $\mathrm{OH}$ with $\mathrm{H}$ or $\mathrm{F}$ in the 2 position of glucose yields analogs that are substrates for transport and hexokinase phosphorylation to the products of DG- and FDG-6-phosphate. Further, these reaction products are not substrates for further metabolism over the time of imaging. It was also demonstrated that the accumulation of DG- and FDG-6-phosphate was in proportion to glycolysis. With ${ }^{11} \mathrm{C}$-labeled glucose, developing an assay is far more complicated because glucose is rapidly metabolized through various pathways, including in the release of ${ }^{11} \mathrm{C}-\mathrm{CO}_{2}$. All this makes it difficult to establish mass conservation of reaction products into a biochemical tracer kinetic assay of glucose metabolism. Mark Raichle and I struggled with all these issues to develop a tracer kinetic model 
for ${ }^{11} \mathrm{C}$-glucose, as shown in the manuscript to which you refer. This tracer kinetic model, however, has a lot of assumptions, and the kinetic imaging was difficult to implement routinely. FDG simplified all this to become the tracer of choice for measuring and imaging glucose metabolism. This illustrates that if a tracer assay is to be adopted in clinical practice it must be reduced to a simple and practical form.

Dr. Lewis: What do you feel are the biggest challenges to nuclear medicine today?

Dr. Phelps: Education. I gave an invited talk at the European Association of Nuclear Medicine meeting last year. As I watched, listened to, and talked with people, I was amazed by the mix of senior and young people, all being excited and ambitious in their lives in nuclear medicine. One reason for this is that nuclear medicine is an independent academic department in most European institutions. They define and control their own destiny. They have their own vision, hopes, and dreams, and there are good jobs for physicians and basic scientists in academia and physicians in practice as a result. In the U.S., we need to get back to training new generations of physicians and basic scientists in the remarkable value and principles of in vivo tracer kinetic imaging assays of biochemistry and biology that are unique to nuclear medicine, molecular imaging, and theranostics. We must be good partners with radiology, the various disciplines in medicine and surgery, and the various disciplines of the basic sciences.

Dr. Lewis: What do you think we can do to bring the best and brightest back into our field?

Dr. Phelps: On the clinical side, radiology gets amazing residents. They're bright young men and women, and, of course, there are jobs for them. I don't think nuclear medicine should be afraid to integrate with the other medical specialties. But both nuclear medicine and radiology have concerns about other specialties taking away their practice. To create the new and growing world we desire for nuclear medicine, we must put this concern aside. At least some academic programs must take the lead to do this. Success solves all problems, and, in this case, mutual success solves all problems.

Success in the clinical sciences and practice produces funding and success in the biomedical sciences that support them, and success in both of these produces success in the industries that support all of them. Molecular imaging of the biology of disease created a pathway to success based on the fundamental principles of nuclear medicine. PET has been driving a success curve for nuclear medicine. Theranostics is the next driver of a growth curve for nuclear medicine and fully integrates molecular imaging diagnostics and molecular therapeutics, consummated with remarkable improvements in patient outcomes, based on a foundation of fundamental principles. This must be supported by academic and commercial research and development (R\&D) to grow the knowledge base and to expand the diversity of indications to benefit patient care.

Dr. Lewis: You mentioned theranostics. Do you feel there's hope or hype?

Dr. Phelps: There is great hope, but, more than that, there are fundamental principles and evidence in improving patient outcomes. Theranostics combines the benefits of conventional drugs and external-beam radiation therapy without some of their limitations and the adverse effects of both. The positive part shared with conventional drugs is that theranostic imaging probes and therapeutics are molecules administered to the patient to search throughout the body with high affinity and specificity for the protein target on cancer cells. They don't, however, have the mass effect of conventional drugs. The positive sharing with externalbeam radiation therapy is the proven and accepted value of radiation therapy; approximately $50 \%$ of cancer patients receive external-beam radiation therapy. The difference is that theranostics provides localized radiation therapy delivered to cancer cells throughout the body that have the target protein, with minimal and manageable side effects.

In teaching theranostics, it is meaningful to ask the students/ audience to focus not only on detecting cancer-it does that — but also on phenotyping cancer cells lesion by lesion throughout the patient's body in the decision to treat or not treat, depending on whether the cancer cells do or do not have the therapeutic protein target. This is personalized medicine in practice. This is amazing and what we've always wanted to do. There is an ever expanding effort academically and commercially to develop biomarkers for phenotyping in blood, biopsy, and surgical samples and through imaging. Theranostics provides an imaging technique that can phenotype cancer cells throughout the body and, with proper use, enrich the percentage of responders in trials to a higher percentage of positive therapeutic outcomes. This should serve as an example of the benefit of phenotyping with molecular imaging for all therapeutics in trials and patient care.

Dr. Lewis: I completely agree. But among the things we have to face as a field is the somewhat, I believe, bogus argument that imaging is too expensive. Do you think we image too much? Do you think that we need to be more selective? If the theranostics paradigm is the way to go, then this has to be done more sensibly and more thoughtfully, so that when we image somebody we do not proceed to treat them if the target is not expressed and visualized.

Dr. Phelps: Agreed. We do image and treat too much within all the things that influence these decisions, as well as the ambiguities that exist in medicine. There will always be ambiguities, but the goal is to reduce them by measurements; you can't improve anything without measurements. As much as possible, we must use good judgment about what we do and don't do that truly improves outcomes for patients, constantly working to produce advances in defining measurements to yield the evidence to support these judgments. This is an endless battle for everyone in biomedical sciences and medicine. In the end, there will always be differences between evidence-based medicine with good judgement and the practicalities of real-world medicine. Our place is to argue the case on evidence-based medicine with good judgment.

Dr. Lewis: Do you think we do enough evidence-based imaging studies?

Dr. Phelps: Certainly not. But, you know, lots of times bad things that happen can produce good outcomes. When x-rays, ultrasound, nuclear medicine, CT, and MRI were introduced, bills were sent and paid. PET moved much later from research to medicine and was faced with the introduction of evidence-based medicine by the Centers for Medicare \& Medicaid Services (CMS). Coverage for PET had to be evidence-based, indication by indication. The Institute for Clinical PET and then in its reformulation into the Academy of Molecular Imaging (AMI) took on this challenge by bringing our colleagues in nuclear medicine, radiology, and various medical and surgical specialties together to engage CMS. We were not making progress until we accepted evidence-based coverage and then came to an agreement with CMS about what evidence CMS would accept. CMS decided they would accept the evidence for many years of clinical research, and then a contractual agreement was formed between AMI and CMS that led to the formation of the National Oncology PET Registry to 
provide independent evidence to accept or reject coverage from trials that CMS and AMI designed together and for which CMS paid for cost recovery for the trials (although portions of the costs were also absorbed by the nuclear medicine programs that performed the trials).

Dr. Lewis: Where do you see nuclear medicine molecular imaging in the next $20 y$ ?

Dr. Phelps: Niels Bohr once said, "Predictions are difficult to make, especially about the future." I think when people predict things, they're often talking about what's happening today. Predictions are, however, intrinsically flawed because there are always unpredictable compensatory responses in extrapolations into the future.

Dr. Lewis: Do you think, for example, there will be another FDG? That there will be another widely accepted approved drug with broad acceptance and use like FDG? Or do you think we're going to just have more niche-type imaging agents that we seem to have now for specific indications?

Dr. Phelps: I think there will be imaging probes with broad applications and that meet the requirements of imaging probes and the assays they provide, although not as broad as FDG-because the broad nature of FDG is the broad importance of glycolysis. Yet, there are controlling biologic alterations that become targets of therapy that span the transitions of many normal biologic processes to those of disease. Although the magnitude of the indications has financial implications in costs to medicine and supporting a field or industry, the low-incident indications remain important to patients with these diseases and to nuclear medicine professionals in the care they provide.

It's interesting that PET research programs built a large library of labeled probes, several thousand of them. Many people ask why they weren't translated to molecular imaging diagnostics. Of course, some of them didn't meet the criteria, but the major reason was that over a long period of time there were no companies supporting PET molecular imaging probe R\&D, along with FDA approval and reimbursement. Commercialization is necessary to have an ongoing pipeline of imaging probes for successful ones to enter medical practice. This industry now exists.

Today things are very different, in that there is a growing academic and industry pipeline with new PET and SPECT molecular imaging probes coming from all over the world. Theranostics is leading the next inflection point in the growth of this pipeline for nuclear medicine, medicine, and patients. I believe pharmaceutical companies are moving from pretending to develop biomarkers and molecular diagnostics to developing them to reduce the cost, time, and number of patients in clinical trials, as well as to reduce the number of patients exposed to the risk of drugs without adding to the benefit the trials seek to achieve. I believe that over time therapy companies will move from the way things are done today to adding biomarkers and molecular diagnostics for the purpose of selecting patients for their drugs. You cannot afford to continue doing things the way they are done now.

Dr. Lewis: I have 2 last questions and a request. What is the 1 question you wish I had asked but didn't?

Dr. Phelps: That's tough. Do I really enjoy and benefit from leading such an unusual and special Department of Molecular \& Medical Pharmacology (DMMP) that integrates in vivo and in vitro (blood) molecular diagnostics and therapy based on the biology of disease that uniquely contains the Ahmanson Translational Imaging Division that contains our nuclear medicine, PET/CT clinical service, and academic programs? I enthusiastically enjoy this because molecular imaging sits at the heart of DMMP, and the department brings together basic scientists across disciplines together with clinical scientists across imaging, medical, and surgical specialties as faculty, students, and staff in a common culture.

Throughout my life, I've tried to do things outside the box with the opportunities this creates and to deal with the problems that originate from it. Defining discoveries and inventions that have the possibility of changing the world for the better originate outside of the box. I have believed for a long time, just as Henry Wagner believed, that the principles of nuclear medicine imaging diagnostics need to be coupled to the principles of pharmacology with common values and the measurements of molecular imaging and other molecular diagnostics to revolutionize therapeutics.

Dr. Lewis: I could not have a conversation with you without you telling me a joke. But please remember that this will be in print. So what's your favorite joke right now?

Dr. Phelps: It's hard to tell jokes today because of the heightened sensitivities. This is a true story rather than a joke. Years ago, I gave a lecture in Dublin, Ireland, with Mikhail Gorbachev. He talked about the breakup of the USSR because of a socialeconomic crisis. Then he talked about his controversial campaign to become the president of Russia, which was also in a socialeconomic crisis. A reporter asked Gorbachev how his campaign was going and to summarize it in 1 word, Gorbachev replied "Good!" The reporter then asked him to summarize it in 2 words. Gorbachev said "Not good!" Gorbachev then explained that when you are in a crisis you must deal with the good and the not good at the same time, and you must keep your sense of humor.

Dr. Lewis: Okay. What's your favorite drink? I have to assume it's an Irish whiskey of some kind. What was your favorite drink of all time?

Dr. Phelps: Single Barrel Jack Daniel's-Tennessee sipping whiskey.

Dr. Lewis: A man after my own heart. I will get you 1 or 2 of those next time I see you! Mike, thank you very much for taking the time to talk to me. Thank you for what you have done for so many of us and our whole field. 